\title{
Rapid within- and transgenerational changes in thermal tolerance and fitness in variable thermal landscapes
}

\author{
Grisel Cavieres ${ }^{1}$, Enrico Rezende ${ }^{1}$, Sabrina Clavijo-Baquet ${ }^{2}$, Jose Alruiz ${ }^{1}$, Carla \\ Rivera-Rebella ${ }^{3}$, Francisca Boher ${ }^{3}$, and Francisco Bozinovic ${ }^{3}$ \\ ${ }^{1}$ Pontificia Universidad Catolica de Chile \\ ${ }^{2}$ Universidad de la República Uruguay \\ ${ }^{3}$ Pontificia Universidad Católica de Chile
}

May 6, 2020

\begin{abstract}
Abstract Phenotypic plasticity allows organisms to cope with variable environmental conditions increasing both performance and fitness. We studied within-generation plasticity and transgenerational effects of thermal conditions on temperature tolerance and demographic parameters in Drosophila melanogaster. We employed a fully factorial design, in which both parental (P) and offspring generations (F1) were reared in a constant or a variable thermal environment. Thermal variability during ontogeny increased heat tolerance in $\mathrm{P}$, but with demographic cost as this treatment resulted in substantially lower survival, fecundity and net reproductive rate. The adverse effects of thermal variability $(\mathrm{V})$ on demographic parameters were less drastic in flies from the F1, which exhibited higher net reproductive rates than their parents. These compensatory responses could not totally overcome the challenges of the thermally variable regime, contrasting with the offspring of flies raised in a constant temperature $(\mathrm{C})$ that showed no reduction in fitness with thermal variation. Thus, the parental thermal environment had effect on thermal tolerance and demographic parameters in fruit-fly. These results demonstrate how transgenerational effects of environmental conditions on heat tolerance, as well as their potential costs on other fitness components, can have a major impact on populations' resilience to warming temperatures and more frequent thermal extremes.
\end{abstract}

\section{Introduction}

Understanding the ecological consequences of changing environments and extreme climatic events has grown more prominent as climate change scenarios predict more frequent and pronounced fluctuations in temperature (Vázquez et al. , 2017). The world's climate is changing dramatically, to such an extent that the 90\% probability interval for global warming from 1990 to 2100 predicts an increase in average temperatures ranging from $1.7{ }^{\circ} \mathrm{C}$ to $4.9{ }^{\circ} \mathrm{C}$ (IPCC (Rahmstorf \& Coumou, 2011)). And while it is acknowledged that warming temperatures will have a significant impact on biodiversity (Gitay et al. , 2002; Meehl \& Tebaldi, 2004; Dawson et al. , 2011; Shukeret al. , 2016), recent studies have shown that the increase in climatic variability and thermal extremes may also have a major impact on populations through a decrease in growth rates, reproduction and survival (Folguera et al. , 2009; Bozinovic et al. , 2016).

Experimental studies have shown that organisms are able to respond to thermal variability through plastic changes in thermal tolerance (Terblanche et al. , 2010; Chidawanyika et al. , 2017). Within-generation plasticity, which include both developmental and reversible plasticity, might impact future generations accelerating the adaptation to novel or fluctuating environments (Ho \& Burggren, 2010; Ezard et al. , 2014). In contrast, transgenerational plasticity refers to phenotypic changes in the offspring generation as a response to environmental inputs experienced by the previous generation (Salinaset al. , 2013; Donelson et al. , 2017). Transgenerational plasticity have been described in several traits, including locomotor performance (Leroi 
et al. , 1994; Seebacher et al. , 2014; Cavieres et al. , 2019), thermal tolerance (Norouzitallab et al. , 2014) and metabolic rate (Donelson et al. , 2012; Le Royet al. , 2017), and enable the offspring to change adaptively according to parental information and avoid the time-lag between environmental signal and phenotypic response (Baker et al. , 2019).

While heat extreme events induce increased tolerance to high temperatures (Bozinovic et al. , 2011; Estay et $a l ., 2011$ ), they may cause organisms to reduce energy allocation to reproduction (Ragland \& Kingsolver, 2008; Roitberg \& Mangel, 2016; Koussoropliset al. , 2017). In this sense, Royama (1992) proposed that the thermal environment can affect demographic parameters through nonlinear changes in fecundity and survival. Thus, high temperatures could negatively impact fitness (Estay et al. , 2011; Clavijo-Baquetet al. , 2014), even if organisms exhibit a seemingly compensatory plastic response in thermal tolerance. Most experimental studies assessing the impact of thermal conditions on animal performance focus on physiological performance, and few studies report those effects in conjunction with Darwinian fitness (Bozinovic et al. , 2011; Nyamukondiwa et al. , 2018).

In this vein, here we quantified within- and transgenerational plasticity of thermal tolerance and demographic parameters in the fruit fly Drosophila melanogaster, and their potential role to ameliorate the negative impact of increased temperature variability. Specifically, we evaluated critical thermal maximum and minimum $\left(C T_{\max }, C T_{\min }\right)$ as indicators of physiological thermal tolerances, and the demographic parameters net reproductive rate (i.e., the average number of offspring produced by an individual during its lifetime, $R_{0}$, ), and generational time (i.e., the average time between the birth of a female and the birth of her first female offspring, $T_{g}$ ) as direct indicators of fitness of flies exposed to variable and constant thermal conditions (Royama, 1992; Pasztor et al. , 1996) (Figure 1).

Overall, we hypothesized that flies reared in variable environments would exhibit a trade-off between physiological and fitness related-traits, namely an increase in heat tolerance but with adverse effects on demographic parameters. We predicted that the negative effects on fitness might be buffered in the subsequent generation if the offspring encountered the same thermal environment as their parents.

\section{Materials and methods}

\section{Experimental setup}

We performed a cross-factorial experiment in which parental flies $(\mathrm{P})$ were raised in constant and variable thermal environments, and their offspring were split and maintained in either the parental environment or the opposite (Figure 1). To obtain this experimental design, we use more than 200 inseminated D. melanogaster collected in central Chile $\left(33^{\circ} 26^{\prime} \mathrm{S}\right.$; $70 \mathrm{deg} 39^{\prime} \mathrm{W}$ at $500 \mathrm{~m}$ above sea level) during 2016 in a nearly $500 \mathrm{~m}^{2}$ habitat. After collection, twenty groups were established with approximately ten females each. Groups were reared in controlled conditions at a constant ambient temperature $\mathrm{T}_{\mathrm{a}}=24 \operatorname{degC}$ and a Light:Dark 12:12 photoperiod. Flies were maintained for three generations in $250 \mathrm{~mL}$ glass vials with Burdick culture medium (Burdick, 1955). Third generation adult males and virgin females from this stock were randomly assigned to two thermal treatments that differ in the variance of temperature. Thermal treatments were $28+-0 \operatorname{deg} \mathrm{C}$ and $28+-4 \operatorname{deg} \mathrm{C}$, a constant (C) and variable (V) thermal environment, respectively, and crossed under these conditions. Acclimation temperatures were chosen based on the well-known limits of fruit fly eggs viability (Hoffmann, 2010) (eggs-to adult viability is $80 \%$ at $28 \mathrm{oC}$ and 0 to $5 \%$ at $32 \mathrm{oC}$, for details see (Hoffmann, 2010; Cavieres et al. , 2018)). In the variable thermal environment, during the day, temperature started to increase linearly at 7:00, reached the maximum at 11:00, then stayed constant, and began to decrease at 19:00 and reached $24 \mathrm{deg} \mathrm{C}$ at 23:00h, the heating/cooling rate between the minimum and maximum temperatures was $0.03 \mathrm{degC} \mathrm{min}^{-1}$ (Figure 1).

The offspring, which corresponds to the parental generation $\mathrm{P}$ in our breeding setup, was maintained from eggs to adult in each thermal treatment. Subsequently, adult males and virgin females from both treatments were evenly divided into two breeding groups and transferred to constant and variable thermal conditions. Their offspring correspond to the F1 in our breeding setup, resulting in a factorial experiment with two $\mathrm{P}$ ( $\mathrm{C}$ and $\mathrm{V}$ for constant and variable, respectively) and four F1 groups (CC, CV, VV and VC, which reflect 
both the parental and offspring thermal environment). As detailed below, we estimated for all $\mathrm{P}$ and $\mathrm{F} 1$ groups the lower and upper thermal critical limits $\left(C T_{\min }\right.$ and $C T_{\max }$, respectively), net reproductive rate $\left(R_{0}\right)$ and generation time $\left(T_{g}\right)$.

\section{Critical thermal limits}

We quantified critical thermal limits in virgin flies 8-10 days old, using the dynamic method (Bozinovic et al. , 2016), which involves heating or cooling flies from a starting temperature until physiological failure (Terblanche et al. , 2007). Flies were placed individually in $5 \mathrm{~mL}$ glass vials into a thermoregulated bath, and the temperature was monitored employing a type $\mathrm{K}$ thermocouple inserted into a control empty vial. The flies were allowed to equilibrate for $10 \mathrm{~min}$ at either 19 or $28 \mathrm{degC}$ before either $C T_{\min }$ or $C T_{\max }$ assessments started, respectively. The cooling and heating rate were $0.1 \operatorname{deg} \mathrm{C} \min \operatorname{deg} \mathrm{C}^{-1}$. We monitored flies every minute and recorded thermal limits as the temperature when postural control was lost. The point of critical thermal minimum $\left(C T_{\min }\right)$ was defined as the temperature of loss of a coordinated muscle function, and critical thermal maximum $\left(C T_{\max }\right)$ was defined as the temperature of onset of muscle spasms as suggested by Terblancheet al. (2007)

\section{Net reproductive rate and generation time}

To quantify ontogenetic and transgenerational effects of thermal environment on $R_{0}$ and $T_{g}$, newly emerged adults from both in $\mathrm{P}$ and $\mathrm{F} 1$ were collected within $8 \mathrm{~h}$ of hatching and transferred to vials containing $6 \mathrm{~g}$ of culture medium. Since temperature may impact fitness-related traits, and that effect could be mediated by population density (Estay et al. , 2011; Royama, 2012; Clavijo-Baquet et al. , 2014), four different population densities were established following the discrete design of Utida (1941) and Royama (1992), which included two, four, eight, and sixteen individuals per vials (sex ratio 1:1). We prepared at least 7 glass vial (cohorts) per density, resulting in a minimum of 28 cohorts per experimental group. Every other day, vials were checked to determine the number of dead flies and to replace the culture medium until complete mortality of the cohort. We then counted the number of eggs from the removed medium to estimate daily fecundity. A Lotka life table (Carey, 2001) was constructed to estimate $R_{0}$ and $T_{g}$ combining data on fecundity $\left(m_{x}\right)$ and the proportion of the surviving individuals at age $x\left(l_{x}\right)$ for each replicate. We estimated $R_{0}$ and $T_{g}$ as $R_{0}=\sum l_{x} m_{x}$ and $t_{g}=\sum x l_{x} \frac{m_{x}}{R_{0}}$.

\section{Statistical analyses}

Before the statistical tests, we evaluated the assumptions of normality and equality of variances using Kolmogorov-Smirnov and Levene tests. To compare critical thermal limits and demographic variables among experimental groups, we included thermal treatment as a factor with six levels (C, V, CV, CC, VC and VV) that describe the thermal experience of flies (direct experience through ontogeny, and indirect thermal experience, through parental thermal exposition; see Figure 1). This factor allowed us to compare phenotypic response between $\mathrm{P}$ and $\mathrm{F} 1$, and also to perform comparisons across $\mathrm{P}$ and $\mathrm{F} 1$ groups. To compare critical thermal limits among experimental groups, we employed linear mixed model with trial as random effect (random intercept) and sex and treatment as predictor variables. Also, to test the potential of trade-off between fitness related traits $\left(R_{0}\right.$ and $T_{g}$ ) and $C T_{\max }$ the one tailed correlation analysis was conducted.

Because the population density effected significantly $R_{0}$ and $T_{g}$ (Supplementary Table S1), we assessed the global response of $R_{0}$ and $T_{g}$ to temperature and density. We performed a nonparametric regression analysis using a generalized additive model (GAM) incorporating populational density (D), parental thermal environment $\left(T_{\mathrm{P}}\right)$, offspring thermal environment $\left(T_{\mathrm{F} 1}\right)$, and thermal treatment (treat $)$ as predictors. We performed GAM since it does not make any a priori assumptions about the shape of relationships between variables, which is key to our evaluation of the effects of population density. Moreover, the main difference between GAMs and linear models is that linear functions of the variables in GAM are replaced by unknown smooth functions, giving additional flexibility to the modeling process (Wood, 2017) . The complexity of the curve (the number of degrees of freedom) and the smoothing terms were determined by penalized regression splines and generalized cross-validation to avoid overfitting (Wood, 2017). Also, we allowed the shrinkage of the smoothers. This technique allows for an extra penalty to be added in the model, and if the penalty is 
high enough, it will shrink all smoothing coefficients to zero. Model selection was done using the AIC criterion $(\triangle \mathrm{AICc}<2$; Burnham \& Anderson (2002)). To perform pairwise comparisons between experimental groups, we performed a posteriori Tukey test following the linear mixed models or GAMs.

All analyses and visualizations were performed in the R statistical environment (http://www.R-project.org/ ). The datasets generated during the current study will be available in the DRYAD repository

\section{Results}

Maximum and minimum critical thermal limits were differentially affected by the thermal environment experienced by flies. $C T_{\max }$ was significantly higher in flies from parental generation reared at variable thermal environment $(\mathrm{V})$ than those reared at constant temperature $(\mathrm{C})$, while $C T_{\min }$ was not different between parental thermal environments (Figures 2A, 2B and Supplementary Figure S1). Interestingly, whereas $C T_{\max }$ was significantly higher in females than males, $C T_{\min }$ did not vary between sex (Table 1, Supplementary Figure S1). With regards to the F1, the thermal environment experienced by parental generation affected the critical thermal limits of their offspring (Table 1, Figure 3). More specifically, F1 flies raised under variable conditions whose parents were also maintained in this environment (i.e., VV) exhibited a significantly higher $C T_{\max }$ than all other $\mathrm{F} 1$ groups (including flies from CV, see Figure 2 and Supplementary Figure S1). Also, $C T_{\max }$ of $\mathrm{F} 1$ flies reared under constant environment, whose parents were reared in the alternative treatment (i.e., VC) had significant lower $C T_{\max }$ than $\mathrm{F} 1$ flies from a constant environment whose parents were maintained in a constant environment (CC). Besides, cold tolerance of $\mathrm{F} 1$ flies reared in a variable thermal environment (i.e., $\mathrm{VV}$ and $\mathrm{CV}$ ) were lower than those reared at $\mathrm{C}$ (i.e., $\mathrm{CC}$ and $\mathrm{VC}$ ).

Changing now to demographic descriptors of fitness, both survival and fecundity per female was lower in $\mathrm{P}$ flies reared at variable environments (Figure 3, detailed analyses in Supplementary Table S2), resulting in substantially lower $R_{0}$ and $T_{g}$ in $\mathrm{C}$ in comparison to V (Figure 2, Supplementary Table S3). In contrast, the variation in survival and fecundity was substantially reduced in the F1 regardless of the thermal treatment, which suggests some sort of compensation across generations (Figures 2 and 3). In fact, flies from the second generation exposed to a variable thermal environment showed a significant increase of $R_{0}$ and $T_{g}$ values compared with their parents (Figure 2). Effects of the parental environment were still evident, however, with both $R_{0}$ and $T_{g}$ being on average lower in F1 lines derived from parents subjected to a variable environment (i.e., VC and VV groups), indicating that the apparent compensatory response to a stressful parental environment was only partial (Figure 2). Interestingly, flies whose parents were maintained at a constant temperature (CC and $\mathrm{CV}$ ) did not show differences in $R_{0}$ and $T_{g}$ between them or their parents regardless of the thermal environment in which they were raised (Figure 2).

Results from our GAM analyses in conjunction with a model comparison approach support the observations listed above (Table 2, Supplementary Table S3). Thermal treatment had a major effect in these fitness components, explaining up to $53.6 \%$ and $34.9 \%$ of the variance in $R_{0}$ and $T_{g}$, respectively, after controlling for density (density effects on demographic variables are represented in Supplementary Figure S2). Furthermore, the models with the lowest AIC in both cases included the treatment factor (see Methods), which encapsulates the thermal environment of the parents $\left(T_{P}\right)$, the offspring $\left(T_{F 1}\right)$ and their interaction, and resulted in a model with a better fit than those where these factors were included separately (Table 1 ).

Interestingly $R_{0}$ and $C T_{\max }$ were negative and significantly correlated $(r=-0.8, T=-2.69, d f=4, P=0.027)$ but, we did not find significant correlations among $T_{g}$ and $C T_{\max }(r=-0.55, T=-1.32, d f=4, P=0.12)$.

\section{Discussion}

Phenotypic plasticity involves phenotypic changes associated with environmental conditions, and may favor the establishment or persistence of organisms in changing environments (Ghalambor et al. , 2007). Consequently, plasticity may potentially affect the selective pressures that a population encounters and, as a result, its evolutionary trajectory (Oster \& Alberch, 1982; Bonduriansky et al. , 2012). Our experiment illustrates how within- and transgenerational plasticity can ameliorate the impact of stressful thermal conditions on physiological and fitness-related traits. In this context, our main results can be summarized as follows. 
First-generation flies subjected to a variable and, stressful thermal environment exhibited higher $C T_{\max }$ when compared against their counterparts maintained at a constant temperature. This plastic and seemingly adaptive response came at costs, however, since these flies also exhibited lower survival rates, fecundity (Figure 3), and, ultimately, $R_{0}$ and $T_{g}$ (Figure 2). Interestingly, these maladaptive plastic responses were less evident in their offspring (Figures 2 and 3), suggesting partial compensation mediated to some degree by transgenerational plasticity.

We aimed to compare the response of the flies under variable and constant thermal environments using both direct measures of fitness and physiological proxies of fitness, such as thermal tolerance, and results were dramatically different (Figure 3). Contrary to results by Nyamukondiwa et al. (Nyamukondiwa et al. , 2018) who evaluated the influence of thermal variability on heat tolerance, our results show that $C T_{\max }$ was positively affected by thermal variability, although with adverse effects on fitness.

These results are intriguing because the temperature peak in the variable thermal environment $\left(32^{\circ} \mathrm{C}\right)$ was substantially lower than the estimated $C T_{\max }\left(\sim 39^{\circ} \mathrm{C}\right)$, and yet this temperature was clearly stressful and impacted survival (Figure 3). Although the impact of temperature peak and time of exposure to thermal extremes on organisms cannot be disentangled, the differences in exposure time might explain this counterintuitive result, since the temperature range that organisms can tolerate is associated with the duration of thermal stress (Rezende et al. , 2014). Consequently, prolonged exposure to high and yet less extreme temperatures elicited an increase in $C T_{\max }$ at a cost in survival and, more importantly, in fecundity rates that are suggestive of a trade-off since less energy could be allocated to reproduction. These results agree with Folguera et al. , (2009) who reported that high environmental thermal amplitude experienced by terrestrial isopods increased the synthesis of stress-inducible heat-shock proteins (HSP), but at a metabolic energy cost with negative effects on longevity and growth rate. Not only is the production of HSP metabolically expensive (e.g., protein biosynthesis represent nearly 30-50\% of total cellular energy consumption(Krebs \& Loeschcke, 1994; Krebs \& Feder, 1997, 1998)), but also they require ATP to maintain the structural integrity of other proteins (Hochachka \& Somero, 2002). In this sense, although plastic responses may mitigate the adverse effects of thermal stress, their compensatory effects might be limited by energetic trade-offs (Pigliucci, 2001; Bozinovic et al. , 2016). Consequently, several studies work with the assumption that higher heat tolerance is a beneficial trait (Cavieres et al. , 2016; Sørensen et al. , 2016; Salachan \& Sørensen, 2017; Salinas et al. , 2019), but here we show that this response was accompanied by a decrease in survival and fecundity, highlighting the importance of incorporating direct measures of fitness in physiological studies in order to have a broad understanding of the implications of phenotypic changes in response to environmental inputs.

Interestingly, fitness cost of living under a variable temperature decreased significantly in the second generation, providing evidence of partial compensation to a stressful thermal environment. This cross-generational compensatory response involves a $133 \%$ increase in $R_{0}$ in the $\mathrm{F} 1$ in comparison to $\mathrm{P}\left(R_{0}=259\right.$ in $\mathrm{VV}$ versus 111 eggs/female in V), but values were still $25 \%$ lower than in flies reared at a constant temperature $\left(R_{0}=\right.$ 325 eggs/female). In this context, our results agree with previous studies that have documented that parental experience modifies the response to environmental input in their offspring. For instance, rapid compensatory responses in tolerance and/or reproductive output have been described in the marine polychaeteOphryotrocha labronica subjected to a low $\mathrm{CO}_{2}$ environment (Rodríguez-Romero et al. , 2016) in Daphnia magna (Gustafsson et al. , 2005) raised with toxic cyanobacteria or coral reef fish Acanthochromis polycanthus (Donelson et al. , 2016) exposed to high temperature (see also(Jensen et al. , 2014; Thor \& Dupont, 2015). Overall, these studies suggest that populations can respond rapidly to pronounced environmental changes, providing putative evidence that non-genetic inheritance might underlie observed responses to rapid changes in climatic conditions (Rando \& Verstrepen, 2007).

The potential impact of selection should not be dismissed, however. Recovery of reproductive output reported in the literature and in our study might result from the synergistic effects of within-generation plasticity and genetic adaptation(Rodríguez-Romero et al. , 2016). As has been recently pointed out, estimates of transgenerational plasticity can be biased due to selection and this is the case even in half- or full-sibs designs(Santos et al. , 2019). Consequently, these effects are particularly relevant in studies dealing with 
responses to stressful environments employing outbred populations (e.g., this study or results for O. labronica (Rodríguez-Romero et al. , 2016)), which is a problem often neglected in the literature of transgenerational effects (Ho \& Burggren, 2010; Burggren, 2014). For instance, the drop in survival and fecundity in P flies from $\mathrm{V}$ may impose strong selection and observed responses in F1 could partly reflect adaptive responses in the Darwinian sense. While the growing evidence indicates that natural populations can respond rapidly to environmental changes, resulting in full or partial compensatory responses to an environmental stress, caution is warranted regarding inferences on the mechanistic basis underlying these responses (Santoset al. , 2019).

To summarize, here we describe how an outbred population of D. melanogaster responds rapidly changing thermal conditions within and across generations. Our analyses provide evidence of a trade-off between thermal tolerance and fitness components such as fecundity in parental flies and pronounced albeit incomplete compensatory responses in their offspring. Similar approaches are necessary to extend studies from withingenerational responses to responses across multiple generations. In this context, we urge future research to be tailored to specific climatic scenarios or geographic regions, aiming to build explanations and predict, in the near future, the potential responses of natural populations to ongoing global warming.

\section{Literature cited}

Baker, D., Sultan Sonia E., Lopez-Ichikawa Maya \& Waterman Robin. 2019. Transgenerational effects of parental light environment on progeny competitive performance and lifetime fitness. Philosophical Transactions of the Royal Society B: Biological Sciences $374: 20180182$.

Bonduriansky, R., Crean, A.J. \& Day, T. 2012. The implications of nongenetic inheritance for evolution in changing environments. Evol Appl 5 : 192-201.

Bozinovic, F., Bastías, D.A., Boher, F., Clavijo-Baquet, S., Estay, S.A. \& Angilletta, M.J. 2011. The Mean and Variance of Environmental Temperature Interact to Determine Physiological Tolerance and Fitness.Physiological and Biochemical Zoology 84 : 543-552.

Bozinovic, F., Medina, N.R., Alruiz, J.M., Cavieres, G. \& Sabat, P. 2016. Thermal tolerance and survival responses to scenarios of experimental climatic change: changing thermal variability reduces the heat and cold tolerance in a fly. Journal of Comparative Physiology B 186 : 581-587.

Burdick, A.B. 1955. Drosophila Experiments for High School Biology. The American Biology Teacher 17 : $155-159$.

Burggren, W.W. 2014. Epigenetics as a source of variation in comparative animal physiology - or - Lamarck is lookin' pretty good these days. The Journal of experimental biology $217: 682-9$.

Burnham, K.P. \& Anderson, D.R. 2002. Model selection and multimodel inference: a practical informationtheoretic approach. Springer Science \& Business Media.

Carey, J.R. 2001. Insect biodemography. Annu. Rev. Entomol.46 : 79-110.

Cavieres, G., Alruiz, J.M., Medina, N.R., Bogdanovich, J.M. \& Bozinovic, F. 2019. Transgenerational and within-generation plasticity shape thermal performance curves. Ecology and Evolution 9 : 2072-2082.

Cavieres, G., Bogdanovich, J.M. \& Bozinovic, F. 2016. Ontogenetic thermal tolerance and performance of ectotherms at variable temperatures. Journal of Evolutionary Biology 29 : 1462-1468.

Cavieres, G., Bogdanovich, J.M., Toledo, P. \& Bozinovic, F. 2018. Fluctuating thermal environments and time-dependent effects on fruit fly egg-hatching performance. Ecology and Evolution 8 : 6849-6851.

Chidawanyika, F., Nyamukondiwa, C., Strathie, L. \& Fischer, K. 2017. Effects of Thermal Regimes, Starvation and Age on Heat Tolerance of the Parthenium Beetle Zygogramma bicolorata (Coleoptera: Chrysomelidae) following Dynamic and Static Protocols. PLoS ONE 12 : e0169371. 
Clavijo-Baquet, S., Boher, F., Ziegler, L., Martel, S.I., Estay, S.A. \& Bozinovic, F. 2014. Differential responses to thermal variation between fitness metrics. Scientific reports 4 : 5349-5349.

Dawson, T.P., Jackson, S.T., House, J.I., Prentice, I.C. \& Mace, G.M. 2011. Beyond Predictions: Biodiversity Conservation in a Changing Climate. Science 332 : 53-58.

Donelson, J., Salinas, S., L. Munday, P. \& Shama, L. 2017. Transgenerational plasticity and climate change experiments: Where do we go from here? Global Change Biology, doi: 10.1111/gcb.13903.

Donelson, J.M., Munday, P.L., McCormick, M.I. \& Pitcher, C.R. 2012. Rapid transgenerational acclimation of a tropical reef fish to climate change. Nature Clim. Change 2 : 30-32.

Donelson, J.M., Wong, M., Booth, D.J. \& Munday, P.L. 2016. Transgenerational plasticity of reproduction depends on rate of warming across generations. Evol Appl 9 : 1072-1081.

Estay, S.A., Clavijo-Baquet, S., Lima, M. \& Bozinovic, F. 2011. Beyond average: an experimental test of temperature variability on the population dynamics of Tribolium confusum. Popul Ecol53 : 53-58.

Ezard, T.H., Prizak, R. \& Hoyle, R.B. 2014. The fitness costs of adaptation via phenotypic plasticity and maternal effects.Functional Ecology 28 : 693-701.

Folguera, G., Bastias, D.A. \& Bozinovic, F. 2009. Impact of experimental thermal amplitude on ectotherm performance: Adaptation to climate change variability? Comparative Biochemistry and Physiology Part A: Molecular $\&$ Integrative Physiology 154 : 389-393.

Ghalambor, C.K., McKay, J.K., Carroll, S.P. \& Reznick, D.N. 2007. Adaptive versus non-adaptive phenotypic plasticity and the potential for contemporary adaptation in new environments. Functional Ecology 21 : 394-407.

Gitay, A., Suarez, A., Watson, R. \& Dokken, J. 2002. Climate Change and Biodiversity. Tech. Rep. IPCC, Geneva, Switzerland.

Gustafsson, S., Rengefors, K. \& Hansson, L.-A. 2005. Increased Consumer Fitness Following Transfer of Toxin Tolerance to Offspring Via Maternal Effects. Ecology 86 : 2561-2567.

Ho, D.H. \& Burggren, W.W. 2010. Epigenetics and transgenerational transfer: a physiological perspective. The Journal of experimental biology 213 : 3-16.

Hochachka, P.W. \& Somero, G.N. 2002. Biochemical Adaptation: Mechanism and Process in Physiological Evolution (Oxford University Press. Inc., ed). New York, NY.

Hoffmann, A.A. 2010. Physiological climatic limits in Drosophila: patterns and implications. The Journal of experimental biology $213: 870-880$.

Jensen, N., Allen, R.M. \& Marshall, D.J. 2014. Adaptive maternal and paternal effects: gamete plasticity in response to parental stress.Functional Ecology 28 : 724-733.

Koussoroplis, A.-M., Pincebourde, S. \& Wacker, A. 2017. Understanding and predicting physiological performance of organisms in fluctuating and multifactorial environments. Ecological Monographs 87 : 178-197.

Krebs, R.A. \& Feder, M.E. 1998. Experimental manipulation of the cost of thermal acclimation in Drosophila melanogaster. Biol J Linn Soc 63 : 593-601.

Krebs, R.A. \& Feder, M.E. 1997. Tissue-specific variation in Hsp70 expression and thermal damage in Drosophila melanogaster larvae. Journal of Experimental Biology 200 : 2007-2015.

Krebs, R.A. \& Loeschcke, V. 1994. Costs and Benefits of Activation of the Heat-Shock Response in Drosophila melanogaster. Functional Ecology 8 : 730-737. 
Le Roy, A., Loughland, I. \& Seebacher, F. 2017. Differential effects of developmental thermal plasticity across three generations of guppies (Poecilia reticulata): Canalization and anticipatory matching.Scientific reports $7: 4313$.

Leroi, A.M., Bennett, A.F. \& Lenski, R.E. 1994. Temperature acclimation and competitive fitness: an experimental test of the beneficial acclimation assumption. Proc. Natl. Acad. Sci. U.S.A.91 : 1917-1921.

Meehl, G.A. \& Tebaldi, C. 2004. More Intense, More Frequent, and Longer Lasting Heat Waves in the 21st Century. Science 305 : 994-997.

Norouzitallab, P., Baruah, K., Vandegehuchte, M., Van Stappen, G., Catania, F., Vanden Bussche, J., et al. 2014. Environmental heat stress induces epigenetic transgenerational inheritance of robustness in parthenogenetic Artemia model. The FASEB Journal 28 : 3552-3563.

Nyamukondiwa, C., Chidawanyika, F., Machekano, H., Mutamiswa, R., Sands, B., Mgidiswa, N., et al. 2018. Climate variability differentially impacts thermal fitness traits in three coprophagic beetle species.PloS one 13 : e0198610.

Oster, G. \& Alberch, P. 1982. Evolution and Bifurcation of Developmental Programs. Evolution 36 : 444-459.

Pasztor, L., Meszena, G. \& Kisdi, E. 1996. R0 or r: A matter of taste? Journal of Evolutionary Biology 9 : $511-516$.

Pigliucci, M. 2001. Phenotypic Plasticity: Beyond Nature and Nurture . JHU Press, Baltimore, Maryland.

Ragland, G.J. \& Kingsolver, J.G. 2008. The effect of fluctuating temperatures on ectotherm life-history traits: comparisons among geographic populations of Wyeomyia smithii. Evol Ecol Res10 : 29-44.

Rahmstorf, S. \& Coumou, D. 2011. Increase of extreme events in a warming world. PNAS 108: 1790517909 .

Rando, O.J. \& Verstrepen, K.J. 2007. Timescales of genetic and epigenetic inheritance. Cell 128: 655-668.

Rezende, E.L., Castaneda, L.E. \& Santos, M. 2014. Tolerance landscapes in thermal ecology. Functional Ecology 28 : 799-809.

Rodriguez-Romero, A., Jarrold, M.D., Massamba-N'Siala, G., Spicer, J.I. \& Calosi, P. 2016. Multigenerational responses of a marine polychaete to a rapid change in seawater pCO2. Evol Appl 9 : 1082-1095.

Roitberg, B.D. \& Mangel, M. 2016. Cold snaps, heatwaves, and arthropod growth. Ecol Entomol 41 : 653-659.

Royama, T. 1992. Analytical population dynamics . Chapman \& Hall, London, United Kingdom.

Royama, T. 2012. Analytical population dynamics . Springer Science \& Business Media.

Salachan, P.V. \& Sorensen, J.G. 2017. Critical thermal limits affected differently by developmental and adult thermal fluctuations. Journal of Experimental Biology 220 : 4471-4478.

Salinas, S., Brown, S.C., Mangel, M. \& Munch, S.B. 2013. Non-genetic inheritance and changing environments. Non-Genetic Inheritance 1 : 38-50.

Salinas, S., Irvine, S.E., Schertzing, C.L., Golden, S.Q. \& Munch, S.B. 2019. Trait variation in extreme thermal environments under constant and fluctuating temperatures. Philos. Trans. R. Soc. Lond., B, Biol. Sci. 374 : 20180177.

Santos, M., Matos, M., Wang, S.P. \& Althoff, D.M. 2019. Selection on structural allelic variation biases plasticity estimates.Evolution 73 : 1057-1062. 
Seebacher, F., Beaman, J. \& Little, A.G. 2014. Regulation of thermal acclimation varies between generations of the short-lived mosquitofish that developed in different environmental conditions. Functional ecology $\mathbf{2 8}$ : 137-148.

Shuker, J.D., Simpkins, C.A. \& Hero, J.-M. 2016. Determining environmental limits of threatened species: the example of the wallum sedgefrog Litoria olongburensis. Ecosphere 7 : n/a-n/a.

Sorensen, J.G., Schou, M.F., Kristensen, T.N. \& Loeschcke, V. 2016. Thermal fluctuations affect the transcriptome through mechanisms independent of average temperature. Scientific Reports 6 : 30975.

Terblanche, J.S., Deere, J.A., Clusella-Trullas, S., Janion, C. \& Chown, S.L. 2007. Critical thermal limits depend on methodological context. Proceedings of the Royal Society of London B: Biological Sciences 274: 2935-2943.

Terblanche, J.S., Nyamukondiwa, C. \& Kleynhans, E. 2010. Thermal variability alters climatic stress resistance and plastic responses in a globally invasive pest, the Mediterranean fruit fly (Ceratitis capitata). Entomologia Experimentalis et Applicata 137 : 304-315.

Thor, P. \& Dupont, S. 2015. Transgenerational effects alleviate severe fecundity loss during ocean acidification in a ubiquitous planktonic copepod. Global change biology 21 : 2261-2271.

Utida, S. 1941. Studies on experimental population of the azuki bean weevil, Callosobruchus chinensis (L.) I. The effect of population density on the progeny populations. Memoirs of the College of Agriculture, Kyoto Imperial University 48 : 1-30.

Vazquez, D.P., Gianoli, E., Morris, W.F. \& Bozinovic, F. 2017. Ecological and evolutionary impacts of changing climatic variability. Biol Rev $92: 22-42$.

Wood, S.N. 2017. Generalized additive models: an introduction with $R$. Chapman and Hall/CRC.

Table 1 Coefficients of the linear mixed model fitted to data for Critical thermal maximum and minimum $\left(C T_{\max }\right.$ and $\left.C T_{\min }\right)$ in Drosophila melanogaster.Significant differences are indicated in bold $(\mathrm{p}<0.05)$. Multiple comparisons in Supplementary Figure S1.

\begin{tabular}{llllll}
\hline Effect & Estimate & SD & DF & T & P \\
\hline CT $_{\max }\left(\mathbf{(}^{\mathbf{o}}\right)$ & & & & & \\
Intercept & 38.8 & 0.15 & 17.1 & 243 & $<\mathbf{0 . 0 0 1}$ \\
V & 0.83 & 0.12 & 329 & 6.81 & $<\mathbf{0 . 0 0 1}$ \\
CC & -0.36 & 0.13 & 315 & -2.78 & $\mathbf{0 . 0 0 5}$ \\
$\mathrm{CV}$ & -0.55 & 0.13 & 313 & -4.08 & $<\mathbf{0 . 0 0 1}$ \\
$\mathrm{VV}$ & 0.55 & 0.14 & 325 & 3.81 & $<\mathbf{0 . 0 0 1}$ \\
VC & -0.78 & 0.13 & 320 & $-5,74$ & $<\mathbf{0 . 0 0 1}$ \\
Male & -0.36 & 0.08 & 315 & $-4,31$ & $<\mathbf{0 . 0 0 1}$ \\
CT $_{\text {min }}\left({ }^{\mathbf{o}} \mathbf{C}\right)$ & & & & & \\
Intercept & 7.78 & 0.09 & 68.0 & 79.2 & $<\mathbf{0 . 0 0 1}$ \\
V & 0.27 & 0.11 & 341 & 2.36 & $\mathbf{0 . 0 2}$ \\
CC & -0.24 & 0.12 & 258 & -1.88 & 0.06 \\
CV & 0.68 & 0.12 & 197 & 6.21 & $<\mathbf{0 . 0 0 1}$ \\
VV & 0.59 & 0.13 & 184 & 4.39 & $<\mathbf{0 . 0 0 1}$ \\
VC & 0.006 & 0.13 & 253 & 0.04 & 0.96 \\
Male & 0.08 & 0.08 & 123 & 1.05 & 0.29 \\
\hline
\end{tabular}

Table 2 Results of the GAM fitted for net reproductive rate $R_{0}$ and generation time $T_{g}$ in Drosophila melanogaster. $D$ is population density, treat is thermal treatment, $T_{\mathrm{P}}$ is parental thermal environment, $T$ F1 is offspring thermal environment. srepresents the cubic regression spline for this variables, Sum edf is the 
sum of effective degrees of freedom; Loglik is log likelihood values, AIC is Akaike information criterion for the model, $\mathrm{r}^{2}$ is determination coefficient. Models with the best fit are shown in bold.

\begin{tabular}{|c|c|c|c|c|}
\hline Model & Sum edf & loglik & $\mathrm{AIC}$ & $\mathrm{r}^{2}$ \\
\hline \multicolumn{5}{|l|}{ Models for $\mathbf{R}_{0}$} \\
\hline$s(D, b y=$ treat $)+$ treat & 5.77 & -1127 & 2279 & 53.6 \\
\hline$s\left(D, b y=T_{\mathrm{P}}\right)+$ treat & 3. 53 & -1131 & 2283 & 51.5 \\
\hline$s\left(D, b y=T_{\mathrm{F} 1}\right)+$ treat & 2.55 & -1137 & 2293 & 48.2 \\
\hline$s(D)+$ treat & 1.41 & -1137 & 2291 & 48.2 \\
\hline \multicolumn{5}{|l|}{ Models for $T_{g}$} \\
\hline $\mathrm{s}(D, b y=$ treat $)+$ treat & 6.09 & -427.3 & 881 & 34.9 \\
\hline $\mathrm{s}\left(D, b y=T_{\mathrm{P}}\right)+$ treat & 3.65 & -438.6 & 898 & 26.5 \\
\hline $\mathrm{s}\left(D, b y=T_{\mathrm{F} 1}\right)+$ treat & 2.53 & -434.2 & 887 & 30.0 \\
\hline $\mathrm{s}(D)+$ treat & 1.74 & -438.2 & 893 & 26.8 \\
\hline
\end{tabular}

\section{Figure legends}

Figure 1. Schematic diagram of the experimental design used to assess within and transgenerational effects of thermal variability on critical thermal limits, and demographic parameters in D. melanogaster . Offspring and parental generation were reared in one of two thermal environments, constant (C) or variable (V), described in the right panel (variable cycles included $8 \mathrm{~h}$ at $24^{\circ} \mathrm{C}, 8 \mathrm{~h}$ at $32^{\circ} \mathrm{C}$ and $8 \mathrm{~h}$ of ramping, see Methods). Abbreviations represent the thermal treatments for the parental generation $(\mathrm{C}$ and $\mathrm{V})$ and the offspring (CC, CV, VV and $\mathrm{VC})$.

Figure 2. Critical thermal limits $\left(C T_{\max }(\mathrm{a})\right.$ and $\left.C T_{\min }(\mathrm{b})\right)$ and demographic parameters $\left(R_{0}(\mathrm{c})\right.$ and $T_{g}(d)$ in our experimental groups. Parental flies $(\mathrm{P})$ and their offspring $(\mathrm{F} 1)$ were reared in a constant $(\mathrm{C}, 28 \pm$ $\left.0^{\circ} \mathrm{C}\right)$ or variable $\left(\mathrm{V}, 28 \pm 4^{\mathrm{O}} \mathrm{C}\right)$ thermal environment. Solid and dashed lines in the right panels represent similar or alternate thermal environments between $\mathrm{P}$ and F1 (see Figure 1). Colored points represent the thermal environment experienced by flies. Values are shown as mean $\pm \mathrm{SE}$.

Figure 3. Survival ( $\mathrm{a}$ and b) and fecundity ( $\mathrm{c}$ and $\mathrm{d}$ ) of $\mathrm{P}$ and $\mathrm{F} 1$ flies of D. melanogaster reared in a constant $\left(\mathrm{C}, 28 \pm 0{ }^{\circ} \mathrm{C}\right)$ or a variable thermal environment $\left(\mathrm{V}, 28 \pm 4{ }^{\circ} \mathrm{C}\right)$. In colors thermal treatments for parental generation (C and V, left panels) and their offspring (CC, CV, VC, VV, right panels). Solid and dashed lines in the right panels represent similar or alternate thermal environments between P and F1 (see Figure 1).

Figure 1

\section{Hosted file}

image1.emf available at https://authorea.com/users/318187/articles/448114-rapid-withinand-transgenerational-changes-in-thermal-tolerance-and-fitness-in-variable-thermallandscapes

Figure 2

\section{Hosted file}

image2.emf available at https://authorea.com/users/318187/articles/448114-rapid-withinand-transgenerational-changes-in-thermal-tolerance-and-fitness-in-variable-thermallandscapes

Figure 3

\section{Hosted file}


image3.emf available at https://authorea.com/users/318187/articles/448114-rapid-withinand-transgenerational-changes-in-thermal-tolerance-and-fitness-in-variable-thermallandscapes

\section{Data accessibility statement}

The datasets analyzed during the current study will be available in the Dryad Digital Repository.

\section{Competing interests}

The authors declare no competing interests

\section{Author contributions}

G.C. and F.Bozinovic designed the experiment. G.C., J.M.A, S.C.B, C.R.R. and F.Boher conducted the experiments. G.C, S.C.B, and E.L.R. analyzed the data, G.C, E.L.R, and F. Bozinovic wrote the paper.

\section{Acknowledgements}

This work was founding by ANID PIA/BASAL [FB0002], FONDECYT [11190637] to G. C., [1190007] to F. Bozinovic, and [1170017] to E.L.R. 